\title{
Charge Photogeneration in Organic Photovoltaics: Role of Hot versus Cold Charge-Transfer Excitons
}

\author{
Bhoj R. Gautam, Robert Younts, Wentao Li, Liang Yan, Evgeny Danilov, Erik Klump, \\ Iordania Constantinou, Franky So, Wei You, Harald Ade, and Kenan Gundogdu*
}

The role of excess excitation energy on long-range charge separation in organic donor/acceptor bulk heterojunctions (BH/s) continues to be unclear. While ultrafast spectroscopy results argue for efficient charge separation through high-energy charge-transfer (CT) states within the first picosecond (ps) of excitation, charge collection measurements suggest excess photon energy does not increase the current density in $\mathrm{BH}$ J devices. Here, the population dynamics of charge-separated polarons upon excitation of high-energy polymer states and low-energy interfacial CT states in two polymer/fullerene blends from ps to nanosecond time scales are studied. It is observed that the charge-separation dynamics do not show significant dependence on excitation energy. These results confirm that excess exciton energy is not necessary for the effective generation of charges.

\section{Introduction}

Despite decades of research, charge photogeneration in polymer-based photovoltaic devices ${ }^{[1-9]}$ is continuing to be a subject of debate. Because of weak dielectric screening ${ }^{[10]}$ in polymers, optical excitations form strongly bound excitons. ${ }^{[11]}$ As a result, charge separation requires a donor/acceptor interface with a large enough driving force to overcome the Coulombic attraction between electrons and holes..$^{[1,4,12,13]}$ It is commonly assumed that excitons first populate interfacial chargetransfer (CT) states, and then separate into free electrons and

Dr. B. R. Gautam, R. Younts, Prof. H. Ade,

Prof. K. Gundogdu

Department of Physics and Organic and Carbon

Electronics Laboratory

North Carolina State University

Raleigh, NC 27695, USA

E-mail: kgundog@ncsu.edu

Dr. W. Li, Dr. L. Yan, Prof. W. You

Department of Chemistry

University of North Carolina at Chapel Hill

Chapel Hill, NC 27599, USA

Dr. E. Danilov

Department of Chemistry

North Carolina State University

Raleigh, NC 27695, USA

E. Klump, I. Constantinou, Prof. F. So

Department of Material Science and Engineering

North Carolina State University

Raleigh, NC 27695, USA

DOI: 10.1002/aenm.201501032 holes. A CT exciton, although formed of partially separated electrons and holes across the interface, is still Coulombically bound. ${ }^{[10,14-19]}$ Moreover, the energy of the exciton at the bottom of the CT band, $\mathrm{CT}_{1}$, is lower compared to the energy of an exciton in the polymer. Hence, an exciton relaxing into the $\mathrm{CT}_{1}$ level loses a significant amount of its original energy, possibly reducing its probability to separate. Indeed, ultrafast studies based on transient absorption, ${ }^{[4]}$ second harmonic generation $(\mathrm{SHG})^{[20]}$ and photoemission spectroscopies, ${ }^{[21]}$ and quantum chemical methods ${ }^{[22-24]}$ have shown that high-energy CT excitons separate very efficiently, suggesting that excess exciton energy is not a waste but is necessary to break the Coulomb bond. On the other hand, time-delayed collection field (TDCF) and highly sensitive external quantum efficiency (EQE) experiments demonstrated that even if an exciton is initially created at $\mathrm{CT}_{1}$, its resulting internal quantum efficiency (IQE) is the same as compared to the IQE of high-energy excitons. ${ }^{\left[{ }^{[9}\right.}$ Hence, there are two contradicting perspectives and explanations of charge separation at a donor/acceptor interface; one involving the need for high-energy (hot) CT excitons ${ }^{[4]}$ and the other showing sufficiency of low-energy (cold) CT excitons. ${ }^{[9]}$

Because excitons created with excess energy quickly thermalize through vibrational energy relaxation, charge separation of hot CT excitons have to be limited to ultrafast time scales, ${ }^{[25]}$ i.e., within the first few 100 fs after excitation. As a result, only ultrafast measurements report charge separation of hot CT excitons. In contrast, TDCF experiments, ${ }^{[26]}$ which are sensitive to the sum of hot and cold exciton separation, measure total charges generated in $10 \mathrm{~ns}$ or more after the excitation. The large difference in the time scales of these two experimental methods introduces challenges to directly compare the results. Here we report optical transient-absorption measurements with ultrafast $\approx 100 \mathrm{fs}$ time resolution and up to $5 \mathrm{~ns}$ total delay after excitation. This time scale almost closes the gap between prior results and allows essential comparison.

In order to study charge separation of hot as well as cold excitons, we performed transient-absorption experiments using two very different conditions. In the first one, the pump photons have high energy and create excitons in the donor polymer with an energy well in excess of even the hot CT state. Probe pulses resonant with hot and relaxed CT transitions and polaron bands measure the CT exciton and polaron population as a function 
of time delay. In this set, both hot and cold charge-separation dynamics are measured. In the second set of experiments, pump photons with energy well below the polymer optical gap and the hot CT state were used. This way, we isolate the cold exciton charge separation by directly populating low-energy CT levels. ${ }^{[9]}$

By analyzing the CT state, polaron, and exciton dynamics in two polymer blends with similar optical properties but different device performance, ${ }^{[27]}$ we found that charge generation is not limited to the first few picoseconds. Both fast charge generation within the fi rst few 100 fs and slow charge generation well beyond the time scales during which excitons would have cooled to the bottom of the CT bands, exist. Moreover, we found that the fast charge generation does not require hot excitons. Even if the lowest-energy CT states are excited directly, an ultrafast charge-generation path still exists in the polymer/ PCBM blends.

\section{Results and Discussion}

We performed transient-absorption spectroscopy (TAS) on blended thin films of phenyl-C61-butyric acid methyl ester (PCBM) and weak donor-strong acceptor medium band gap copolymers $(\mathrm{PBnDT}-\mathrm{XTAZ} \text {, where } \mathrm{X}=\mathrm{F} \text { or } \mathrm{H})^{[28]}$ with weight ratio 1:2. These polymers have benzodithiophene (BnDT) moiety as the donor and benzotriazole (HTAZ) or fluorinated benzotriazole (FTAZ) as the acceptor. Depending on fluorination of the TAZ moiety, they are commonly named as PBnDTFTAZ and PBnDT-HTAZ. Other than the fluorine substitution, they have the same molecular structure, resulting in very similar steady-state absorption spectra (see Figure1). Namely, the vibronic features and the onset of optical absorption have the same energies for both polymers. The fluorine substitution leads to slight differences in aggregation, which is reflected in the relative intensities of the $0-0$ and $0-1$ vibronic transitions in the absorption spectra ${ }^{[29]}$ and molecular packing, i.e., propensity for $\pi-\pi$ stacking, as observed in X-ray diffraction spectroscopy. ${ }^{[28]}$ These materials are well studied, and differences in device performance have been attributed to differences in

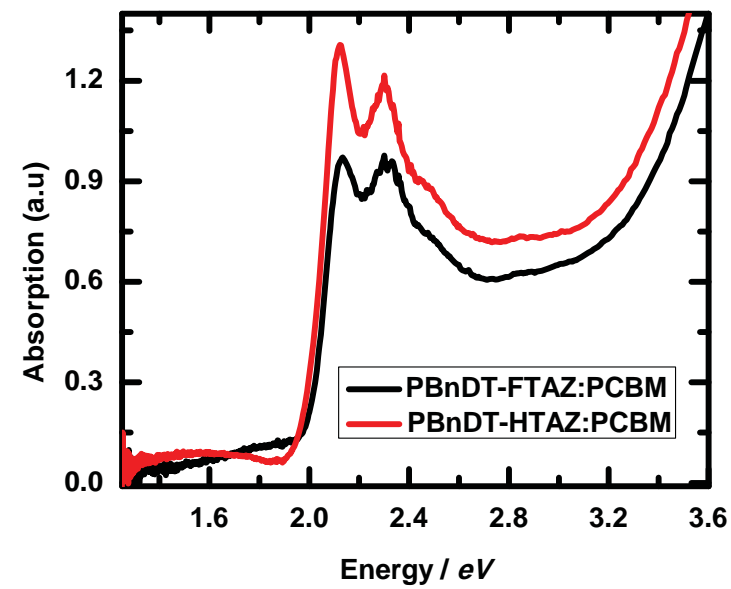

Figure 1. Absorption spectra of PBnDT-FTAZ:PCBM and PBnDTHTAZ:PCBM films.

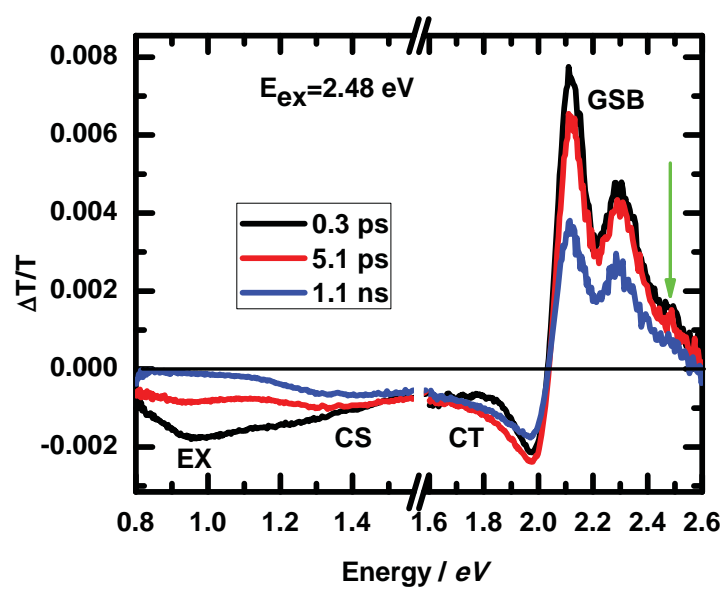

Figure 2. Room temperature TAS for PBnDT-FTAZ:PCBM with a pump excitation of $2.48 \mathrm{eV}$ marked by the green arrow. GSB stands for "groundstate bleaching." In the IR region: EX peak is due to excited-state absorption of polymer excitons, CS is due to absorption by polarons. In the visible region, CT marks the PIA due to charge-transfer excitons.

mobilities and not charge-generation efficiencies. ${ }^{[27]}$ Hence they provide a set of interesting complementary systems to study charge-generation dynamics as a function of excitation energy.

Figure 2 shows transient spectra of the FTAZ-blended films at three different pump and probe delays $(0.3 \mathrm{ps}, 5.1 \mathrm{ps}$, and $1.1 \mathrm{~ns})$. Here the pump photon energy $(2.48 \mathrm{eV})$ produces above-gap excitations. The pump power is adjusted to have an initial photoexcitation density of $4 \times 10^{16} \mathrm{~cm}^{-3}$. In the IR region, two spectrally broad bands centered near 1 and 1.2-1.4 eV are apparent. These features were respectively assigned to absorption by singlet excitons and polarons (charge separated states) based on earlier spectroscopy studies in blends of various polymers $^{[4,6,30-32]}$, and doping-induced absorption spectra (Figure S1, Supporting Information). This assignment is further confirmed with the evolutions of the features in the neat (Figure S2, Supporting Information) and blended samples. While the exciton peak dominates the IR response in the neat polymer (Figure S3, Supporting information), in the blended thin films, the exciton peak vanishes quickly and the polaron peak dominates the IR transient spectra.

In the visible range, the vibronic structure above $2 \mathrm{eV}$ is due to the bleaching of the ground state. The photoinduced absorption (PIA) band at 1.6-1.8 eV is due to the CT excitons. This assignment is based on the elimination of other sources as possibility and comparison with the TAS of neat thin films. The singlet exciton at $\approx 1 \mathrm{eV}$ decays much faster and there is no rise in PIA signal at this band for neat films (Figure S4, Supporting Information). In contrast, blended films show a rise in transient signal in the first $100 \mathrm{ps}$ due to formation of CT excitons. The assignment is also in agreement with spectroscopic studies of a similar band gap $(1.9 \mathrm{eV})$ thiophene-based polymer (P3HT) blend published in the literature. ${ }^{[6]}$ Previous studies on the blends of a variety of low and medium band gap polymer materials such as PCPDTBT:PCBM, PTB7:PCBM, series of PTBF blends, and P3HT:PCBM ${ }^{[30,31,33]}$ show that the spectral position of the PIA band due to CT excitons is within $1.2-1.95 \mathrm{eV}$. 

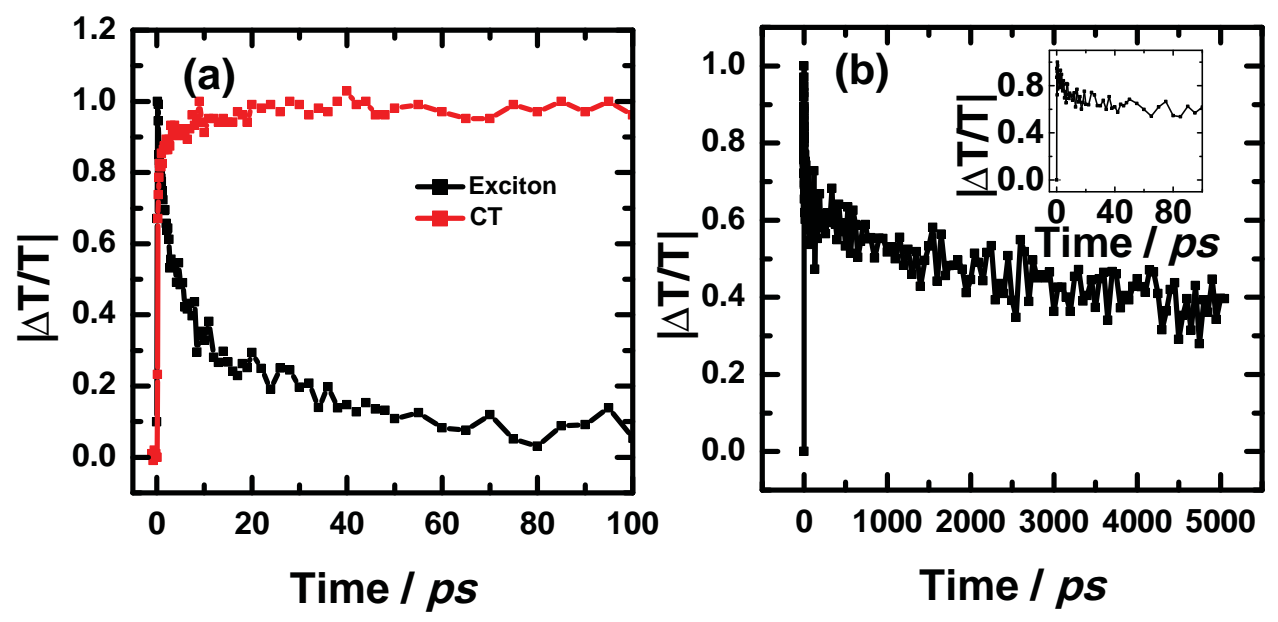

Figure 3. Normalized dynamics of a) CT exciton and singlet exciton PIA and b) polaron acquired at room temperature for PBnDT-FTAZ:PCBM at pump excitation $2.48 \mathrm{eV}$. Inset in part (b) shows the early dynamics within $100 \mathrm{ps}$.

In order to measure the time evolution of excitons and polarons, we isolate individual contributions of these species to the IR region of the spectra by deconvolution. The details of this procedure are described elsewhere ${ }^{[30,31]}$ and summarized in Figure S5 in the Supporting Information. Figure 3a shows the singlet exciton decay of FTAZ blend plotted together with the rise of the CT exciton population as extracted from the evolution of the $1.71 \mathrm{eV}$ feature. The exciton decay is characterized by 3 and $40 \mathrm{ps}$ time constants, while the CT exciton population exhibits an immediate rise ( $\approx$ hundreds of fs) followed by a slower rise, i.e., 8 ps characteristic time. These CT excitons then decay slowly, characterized by 484 ps and 16.3 ns time constants. The difference in the decay of the exciton and the rise of CT exciton population suggests that, while the primary loss of exciton population is due to transfer to the CT states, it is not the only depopulation process.

Given that FTAZ devices are very efficient, the vast majority of excitations must lead to CT states, if direct creation of charges is assumed to be not occurring. The time evolution of the polaron peak, on the other hand, does not correlate with the decay of the CT exciton and the exciton features. The polaron peak is substantially stronger at the beginning (Figure $3 \mathrm{~b}$ ) and then decays over time with characteristic times of $11 \mathrm{ps}$ and $>5 \mathrm{~ns}$. The immediate appearance of the polaron peak within the instrument response time suggests that indeed there is charge generation on ultrafast time scales.

The immediate appearance of a polaron spectral signature may suggest charge generation through hot CT exciton states prior to relaxation to the bottom of the CT band. In order to check this possibility, we performed a second set of ultrafast experiments with pump pulses at $1.28 \mathrm{eV}$, which is below the optical gap of the polymer ${ }^{[28,34]}$ and fullerene constituents, ${ }^{[34]}$ below the hot CT state, and resonant with the lowest energy CT states, $\mathrm{CT}_{1}$, as determined using electroluminescence measurements (Figure S6, Supporting Information). ${ }^{[9,35]}$ Figure 4a shows the resulting TAS spectra at three different delays. The inset in Figure $4 \mathrm{~b}$ shows the in-pulse formation of CT excitons. The differences in the CT exciton formation in above and below-gap excitation are due to the fact that
$\mathrm{CT}_{1}$ is populated directly with $1.28 \mathrm{eV}$ photons in contrast to above-gap excitation where singlet excitons are formed first in polymer domain, which decay into CT state at later time. With the $1.28 \mathrm{eV}$ excitation, the polaron peak immediately forms (Figure 4c), similar to what has been observed in the abovegap excitation, and dominates the spectra with highly suppressed singlet exciton generation (Figure 4a). We performed intensity-dependent measurements to confirm that observed polaron generation is due to CT excitation but not due to excitons created by two photon absorptions. The intensity of the polaron peak exhibits strong correlation with the CT exciton peak and it is not correlated with the exciton-peak intensity (Figure S7, Supporting Information). This suggests the primary source of the polarons in below-gap excitation is the CT excitation. Since the polaron population evolves similarly in both below and above-gap excitation, the immediate charge generation on the ultrafast time scales does not have a significant correlation with the excitation energy. Even at low excitation energies, polarons form in the first few $100 \mathrm{fs}$. Therefore, hot energy exciton charge separation is neither the primary source for charge generation nor it is necessary for effective charge splitting. Even if the energy of the initial CT exciton is very low, it can separate very quickly into polarons. This is further supported by the EQE results shown in Figure S8 in the Supporting Information. These findings seem to be in line with energy considerations and kinetic arguments presented recently ${ }^{[36]}$ as well as the results of some recent experiments utilizing below-gap excitation. ${ }^{[9,16,37]}$

We performed similar experiments in HTAZ blends. The results are summarized in Figure 5. Again, regardless of excitation energy below or above the hot CT state, there is an immediate charge population created within the instrument response of the experiment, further confirming that hot CT states do not play a significant role in separation of charges.

Comparison of HTAZ and FTAZ data provides further information about the initial steps of charge generation and the role of fluorination in these blends. One surprising result is for above-gap excitation, the characteristic rise times of the CT population are exactly the same for both blends, i.e., 

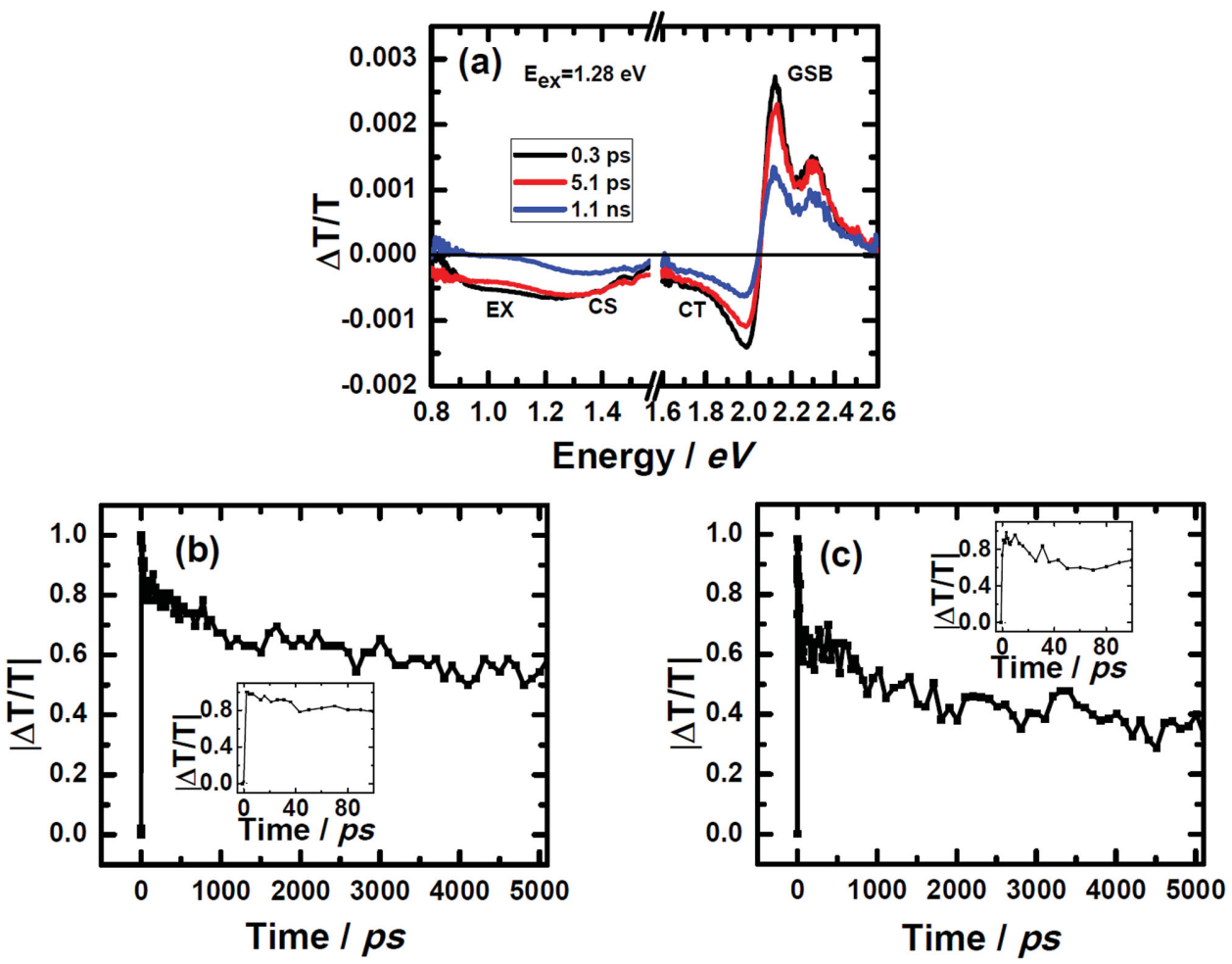

Figure 4. a) TAS and normalized dynamics of b) $\mathrm{CT}$ and c) polaron acquired at room temperature for PBnDT-FTAZ:PCBM at pump excitation energy of $1.28 \mathrm{eV}$. Exciton band in (a) is highly suppressed when compared to above-gap excitation (Figure 2). Insets in (b) and (c) show the immediate CT and charge generation.

8 ps, indicating that the fluorination does not change the CT formation rate (Figure 5c). Similar time constants have been observed in P3HT:PCBM system. ${ }^{[6]}$ At first sight, this is somewhat unexpected because CT exciton formation is related to exciton diffusion to the interfaces. As morphological characteristics show, larger domain spacing and low crystallinity in TAZ blends in contrast to the prototypical P3HT blends, ${ }^{[12,38]}$ one would expect lower CT formation rates for the TAZ blends. However, it is well established by now that fullerenes are miscible in the amorphous portions of the polymer ${ }^{[39-41]}$ and that devices have large volume fractions of mixed domains with molecular distribution, rather than discrete interfaces. ${ }^{[27]}$

In contrast to the rise of the CT population, the decay dynamics shows differences. The FTAZ blend exhibits significantly faster relaxation of the CT state compared to the HTAZ blend (Figure 5c). There could be two potential explanations for faster CT population decay in FTAZ; either CT excitons efficiently separate and depopulate the CT levels or they efficiently recombine in comparison to HTAZ. The latter is less likely because fluorination is known to reduce the CT exciton binding ${ }^{[42]}$ due to increased electron-hole chargetransfer complex separation. ${ }^{[28]}$ Hence, the charge separation should be dominating the relaxation of the CT band. The fact that the CT decay is taking place in several nanoseconds, a time period much longer than vibrational energy relaxation of high-energy excitons, again supports that the chargegeneration pathway is mostly taking place from the cold CT states. ${ }^{[9]}$ Moreover, FTAZ and HTAZ blends show the same external charge-generation efficiency $(\mathrm{EGE})^{[27]}$ despite the differences in the $\mathrm{CT}$ exciton decay dynamics. The difference in dynamics in the face of the same EGE simply implies that caution should be used when interpreting dynamic data in the context of organic photovoltaics (OPV) devices. Longer CT exciton dynamics does not necessarily mean that charges get lost. Sensitive IQE measurement on OPVs based on these blends can determine the relative contribution of cold and hot CT states to photocurrent.

Comparison of the decay of the polaron feature at $1.3 \mathrm{eV}$ also provides information about the dynamics of the charges after they are separated. For HTAZ blend, the polaron peak rises slightly within the first few $100 \mathrm{ps}$ and then decays. The characteristic rise time for charge generation is $10 \mathrm{ps}$ and decay time is $15.6 \mathrm{~ns}$ ( $>5 \mathrm{~ns}$ time window) in HTAZ blend. On the other hand, the FTAZ blend shows the immediate rise within the first ps and then decays with $11 \mathrm{ps}$ and $9.1 \mathrm{~ns}$ ( $>5 \mathrm{~ns}$ time window) time constants. In FTAZ blends, the polaron population relaxes at a faster rate compared to HTAZ blend (Figure 5d, f) at longer delay times. At longer periods, nongeminate recombination (bimolecular) leads to decay of the polaron population. Bimolecular recombination is only possible if the holes in polymer domains are mobile enough to encounter electrons in the fullerene. Recent work on these blends suggests that the major reason for the difference in the performance of OPVs based on these two polymers is the higher hole mobility in FTAZ, leading to higher charge extraction. ${ }^{[27]}$ This is in agreement with our results. Since charges are not extracted in the current experiments, which are effectively conducted at $V_{\mathrm{OC}}$ conditions, higher hole mobility in 

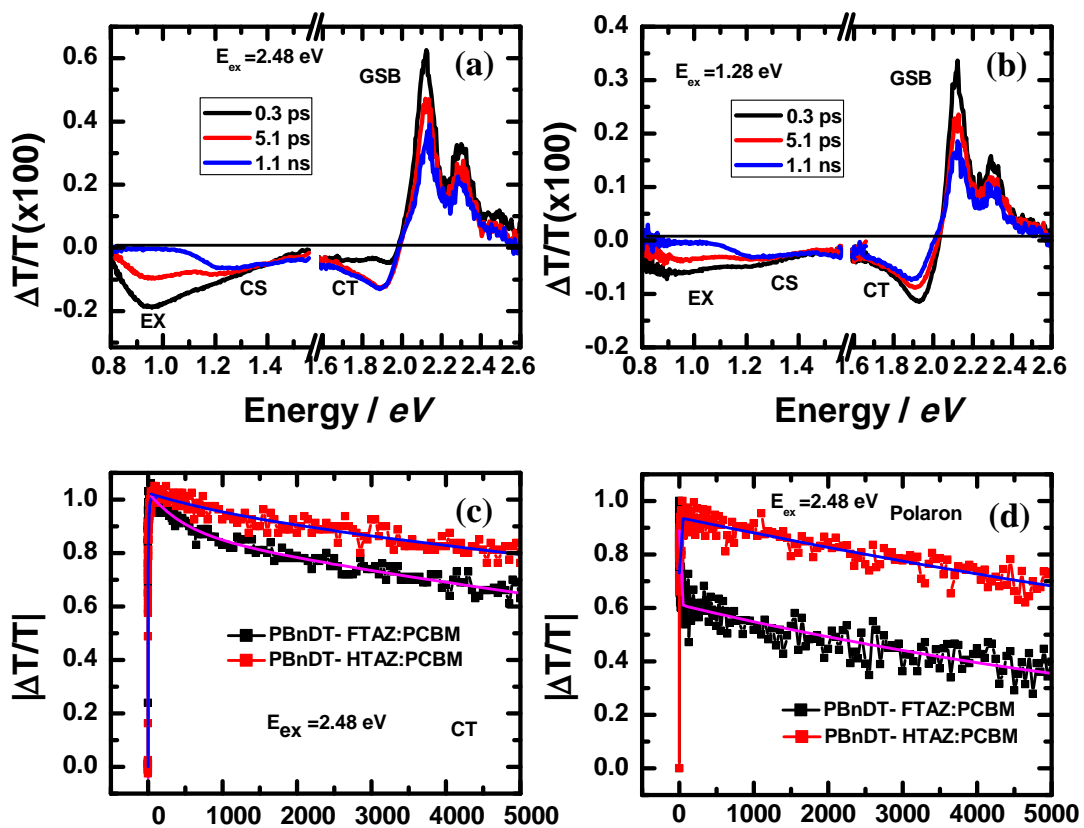

Time / ps
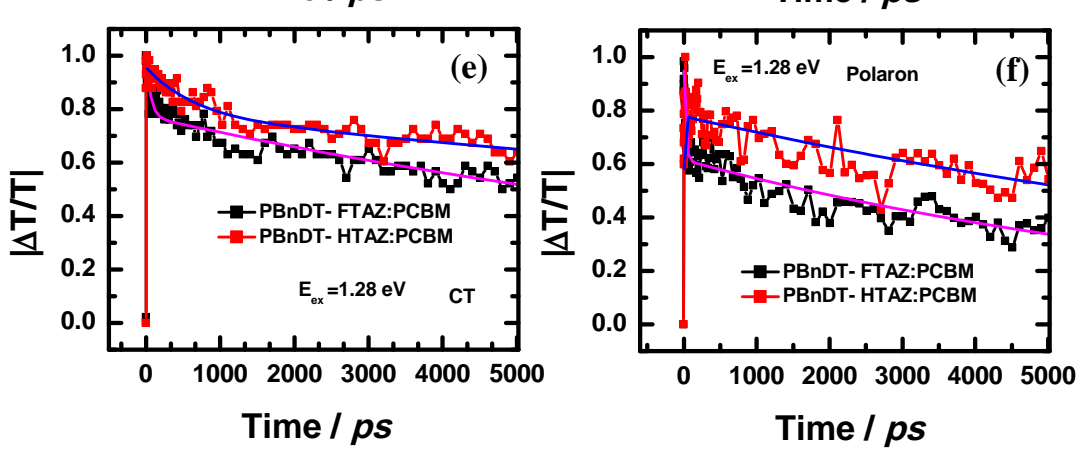

Figure 5. a,b) TAS for PBnDT-HTAZ:PCBM (1:2) at pump excitation of $2.48 \mathrm{eV}$ and $1.28 \mathrm{eV}$, respectively. $\mathrm{c}, \mathrm{e})$ Comparison of charge-transfer dynamics of PBnDT-FTAZ:PCBM and PBnDT-HTAZ:PCBM at $2.48 \mathrm{eV}$ and $1.28 \mathrm{eV}$ excitation, respectively. $\mathrm{d}, \mathrm{f}$ ) Comparison of polaron dynamics of PBnDT-FTAZ:PCBM and PBnDT-HTAZ:PCBM at $2.48 \mathrm{eV}$ and $1.28 \mathrm{eV}$ excitation, respectively. and CT exciton population growth rates. On the other hand charge separation of CT excitons is faster in fluorinated polymer.

\section{Experimental Section}

Sample Preparation: Blends of PBnDT-(X) TAZ:PCBM (1:2 w/w; $12 \quad \mathrm{mg} \mathrm{mL}^{-1}$ for FTAZ and $7.5 \mathrm{mg} \mathrm{mL}^{-1}$ for HTAZ) were dissolved in 1,2,4-trichlorobenzene with heating at $120-140^{\circ} \mathrm{C}$ for $6 \mathrm{~h}$. The molecular weights of FTAZ and HTAZ were 30 and $40 \mathrm{~kg} \mathrm{~mol}^{-1}$, respectively. Blend films were prepared by spin casting the hot solution on glass substrates at $500 \mathrm{rpm}$ for $60 \mathrm{~s}$. The glass substrates were cleaned ultrasonically using deionized water, acetone, and isopropanol for $15 \mathrm{~min}$ per cleaning solvent before spin casting. Neat FTAZ films were prepared using same procedure from $14 \mathrm{mg} \mathrm{mL}^{-1}$ of solution. The substrates were transferred into vacuum chamber immediately after spin-coating and then dried at reduced pressure of $5 \mathrm{~mm} \mathrm{Hg}$ for $30 \mathrm{~min}$.

Measurements: A Cary win 50 UV-spectrophotometer from Varian was used for the roomtemperature ground-state absorption measurement. Transient-absorption data were collected using TAS setup. This setup consists of the spectrometer (Ultrafast Helios system) and amplified Ti:Saphhire Laser. The output of amplified Ti:Saphhire laser provides $800 \mathrm{~nm}$ fundamental pulses at $1 \mathrm{kHz}$ repetition rate, which were split into two optical beams to generate pump and probe pulses. One fundamental beam was used to generate pump beam using an optical parametric amplifier system (Coherent Opera Solo). A white light/NIR probe was generated by focusing another fundamental beam into a sapphire plate/flint glass. Pump and probe beams were focused on a sample and probe light was collected by a charge-coupled device (CCD). The spectral detection region is $0.8-2.6 \mathrm{eV}$. The thin film samples were encapsulated using UV curable clue before measurement. Samples were robust in this environment, which was confirmed by unchanged kinetics over multiple scans. The instrument response function was $\approx 100 \mathrm{fs}$ full width at half maximum (FWHM).
FTAZ domains should lead to enhanced bimolecular recombination in comparison to HTAZ blend.

\section{Conclusions}

In conclusion, we studied charge-transfer exciton and polaron dynamics in two optically comparable polymer blends. Our results imply that the charge-separation dynamics and chargegeneration efficiency do not depend strongly on excitation energy. Regardless of the excitation energy, there exists an ultrafast kinetic component in charge separation. Since charges separate in similar rates regardless of the initial exciton energy being above the polymer singlet levels or in lowest CT states, photons with energy equal or higher than $\mathrm{CT}_{1}$ can produce the same charge-generation yield. Moreover, we found that fluorination does not have an impact on singlet exciton quenching

\section{Supporting Information}

Supporting Information is available from the Wiley Online Library or from the author.

\section{Acknowledgements}

This work was supported by Office of Naval Research (ONR) grant N000141310526 P00002 (B.R.G, R.Y, and K.G.), ONR grant N000141410531 (H.A.), ONR grant N000141410221 (W.L. and W.Y.), NSF SNM grant ECCS-1344745 (L.Y. and W.Y.), and ONR grant N000141110245 (E.K., I.C., and F.S.). The authors acknowledge Steffen Roland and Prof. Dieter Neher for the electroluminescence measurements.

Received: May 25, 2015 Revised: September 3, 2015 Published online: October 26, 2015 
[1] A. A. Bakulin, A. Rao, V. G. Pavelyev, P. H. van Loosdrecht, M. S. Pshenichnikov, D. Niedzialek, J. Cornil, D. Beljonne, R. H. Friend, Science 2012, 335, 1340.

[2] S. R. Cowan, N. Banerji, W. L. Leong, A. J. Heeger, Adv. Funct. Mater. 2012, 22, 1116.

[3] S. Gélinas, A. Rao, A. Kumar, S. L. Smith, A. W. Chin, J. Clark, T. S. van der Poll, G. C. Bazan, R. H. Friend, Science 2014, 343, 512.

[4] G. Grancini, M. Maiuri, D. Fazzi, A. Petrozza, H. Egelhaaf, D. Brida, G. Cerullo, G. Lanzani, Nat. Mater. 2013, 12, 29.

[5] J.-L. Brédas, J. E. Norton, J. Cornil, V. Coropceanu, Acc. Chem. Res. 2009, 42, 1691.

[6] S. Singh, B. Pandit, T. P. Basel, S. Li, D. Laird, Z. V. Vardeny, Phys. Rev. B 2012, 85, 205206.

[7] L. G. Kaake, J. J. Jasieniak, R. C. Bakus, G. C. Welch, D. Moses, G. C. Bazan, A. J. Heeger, J. Am. Chem. Soc. 2012, 134, 19828.

[8] M. Tong, N. E. Coates, D. Moses, A. J. Heeger, S. Beaupré, M. Leclerc, Phys. Rev. B 2010, 81, 125210.

[9] K. Vandewal, S. Albrecht, E. T. Hoke, K. R. Graham, J. Widmer, J. D. Douglas, M. Schubert, W. R. Mateker, J. T. Bloking, G. F. Burkhard, A. Sellinger, J. M. J. Fréchet, A. Amassian, M. K. Riede, M. D. McGehee, D. Neher, A. Salleo, Nat. Mater. 2014, $13,63$.

[10] D. Niedzialek, I. Duchemin, T. B. de Queiroz, S. Osella, A. Rao, R. Friend, X. Blase, S. Kümmel, D. Beljonne, Adv. Funct. Mater. 2015, 25, 1972.

[11] O. G. Reid, R. D. Pensack, Y. Song, G. D. Scholes, G. Rumbles, Chem. Mater. 2013, 26, 561.

[12] Y. Zhang, T. P. Basel, B. R. Gautam, X. Yang, D. J. Mascaro, F. Liu, Z. V. Vardeny, Nat. Commun. 2012, 3, 1043.

[13] S. M. Falke, C. A. Rozzi, D. Brida, M. Maiuri, M. Amato, E. Sommer, A. De Sio, A. Rubio, G. Cerullo, E. Molinari, C. Lienau, Science 2014, $344,1001$.

[14] C. Deibel, T. Strobel, V. Dyakonov, Adv. Mater. 2010, 22, 4097.

[15] F. Deschler, E. Da Como, T. Limmer, R. Tautz, T. Godde, M. Bayer, E. von Hauff, S. Yilmaz, S. Allard, U. Scherf, J. Feldmann, Phys. Rev. Lett. 2011, 107, 127402.

[16] J. Lee, K. Vandewal, S. R. Yost, M. E. Bahlke, L. Goris, M. A. Baldo, J. V. Manca, T. V. Voorhis, J. Am. Chem. Soc. 2010, 132, 11878.

[17] M. C. Scharber, C. Lungenschmied, H.-). Egelhaaf, G. Matt, M. Bednorz, T. Fromherz, J. Gao, D. Jarzab, M. A. Loi, Energy Environ. Sci. 2011, 4, 5077.

[18] D. Veldman, S. C. Meskers, R. A. Janssen, Adv. Funct. Mater. 2009, 19, 1939

[19] X.-Y. Zhu, Q. Yang, M. Muntwiler, Acc. Chem. Res. 2009, 42, 1779.
[20] M. Schulze, M. Hänsel, P. Tegeder, J. Phys. Chem. C 2014, 118, 28527.

[21] A. E. Jailaubekov, A. P. Willard, J. R. Tritsch, W.-L. Chan, N. Sai, R. Gearba, L. G. Kaake, K. J. Williams, K. Leung, P. J. Rossky, X-Y. Zhu, Nat. Mater. 2013, 12, 66.

[22] X. Shen, G. Han, D. Fan, Y. Xie, Y. Yi, J. Phys. Chem. C 2015, 119, 11320.

[23] M. Huix-Rotllant, H. Tamura, I. Burghardt, J. Phys. Chem. Lett. 2015, 6, 1702.

[24] H. Tamura, I. Burghardt, J. Am. Chem. Soc. 2013, 135, 16364.

[25] Y. Song, S. N. Clafton, R. D. Pensack, T. W. Kee, G. D. Scholes, Nat. Commun. 2014, 5, 4933.

[26] J. Kniepert, M. Schubert, J. C. Blakesley, D. Neher, J. Phys. Chem. Lett. 2011, 2, 700.

[27] W. Li, S. Albrecht, L. Yang, S. Roland, J. R. Tumbleston, T. McAfee, L. Yan, M. A. Kelly, H. Ade, D. Neher, W. You, J. Am. Chem. Soc. 2014, 136, 15566.

[28] S. C. Price, A. C. Stuart, L. Yang, H. Zhou, W. You, J. Am. Chem. Soc. 2011, 133, 4625.

[29] F. C. Spano, Acc. Chem. Res. 2010, 43, 429.

[30] B. S. Rolczynski, J. M. Szarko, H. J. Son, L. Yu, L. X. Chen, J. Phys. Chem. Lett. 2014, 5, 1856.

[31] J. M. Szarko, B. S. Rolczynski, S. J. Lou, T. Xu, J. Strzalka, T. J. Marks, L. Yu, L. X. Chen, Adv. Funct. Mater. 2014, 24, 10.

[32] J. Guo, H. Ohkita, H. Benten, S. Ito, J. Am. Chem. Soc. 2010, 132, 6154.

[33] G. Grancini, D. Polli, D. Fazzi, J. Cabanillas-Gonzalez, G. Cerullo, G. Lanzani, J. Phys. Chem. Lett. 2011, 2, 1099.

[34] M. Hallermann, I. Kriegel, E. Da Como, J. M. Berger, E. Von Hauff, J. Feldmann, Adv. Funct. Mater. 2009, 19, 3662.

[35] K. Tvingstedt, K. Vandewal, A. Gadisa, F. Zhang, J. Manca, O. Inganas, J. Am. Chem. Soc. 2009, 131, 11819.

[36] X.-Y. Zhu, J. Phys. Chem. Lett. 2014, 5, 2283.

[37] A. Zusan, K. Vandewal, B. Allendorf, N. H. Hansen, J. Pflaum, A. Salleo, V. Dyakonov, C. Deibel, Adv. Energy. Mater. 2014, 4, 1400922.

[38] J. R. Tumbleston, A. C. Stuart, E. Gann, W. You, H. Ade, Adv. Funct. Mater. 2013, 23, 3463.

[39] B. A. Collins, E. Gann, L. Guignard, X. He, C. R. McNeill, H. Ade, J. Phys. Chem. Lett. 2010, 1, 3160.

[40] N. D. Treat, M. A. Brady, G. Smith, M. F. Toney, E. J. Kramer, C. J. Hawker, M. L. Chabinyc, Adv. Energy. Mater. 2011, 1, 82.

[41] B. Watts, W. J. Belcher, L. Thomsen, H. Ade, P. C. Dastoor, Macromolecules 2009, 42, 8392.

[42] P. Yang, M. Yuan, D. F. Zeigler, S. E. Watkins, J. A. Lee, C. K. Luscombe, J. Mater. Chem. C 2014, 2, 3278. 